S. BÜYÜKÖZTÜrK ${ }^{1}$, Ç. KeKIK ${ }^{2}$, A.Z. GÖKYIĞIT ${ }^{3}$, F.İ. TeZER FILIK ${ }^{41}$, G. KARAKAYA ${ }^{4}$,

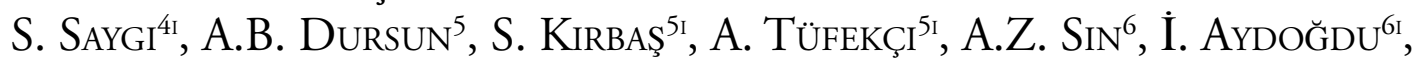
M.H. Sorgun ${ }^{71}$, N. Aydin ${ }^{71}$, A. GelinciK ${ }^{1}$, B. ÇOlaKoĞLU ${ }^{1}$, G. ÇeliK ${ }^{7}$, F. OĞUZ ${ }^{2}$

\title{
Cutaneous drug reactions to antiepileptic drugs and relation with HLA alleles in the Turkish population
}

\author{
${ }^{1}$ Istanbul University, Istanbul Faculty of Medicine, Department of Internal Medicine, Allergy and Clinical Immunology Unit, \\ Istanbul, Turkey \\ ${ }^{2}$ Istanbul University, Istanbul Faculty of Medicine, Department of Medical Biology, Istanbul, Turkey \\ ${ }^{3}$ Istanbul University, Istanbul Faculty of Medicine, Department of Neurology, Istanbul, Turkey \\ ${ }^{4}$ Hacettepe University, Faculty of Medicine, Department of Chest Medicine, Immunology and Clinical Immunology Unit, Ankara, Turkey \\ ${ }^{4}$ Hacettepe University, Faculty of Medicine, Department of Neurology, Ankara, Turkey \\ ${ }^{5}$ Recep Tayyip Erdoğan University, Faculty of Medicine, Department of Internal Medicine, Allergy and Clinical Immunology Unit, \\ Rize, Turkey \\ ${ }^{5 i}$ Recep Tayyip Erdoğan University, Faculty of Medicine, Department of Neurology, Rize, Turkey \\ ${ }^{6}$ Ege University, Faculty of Medicine, Department of Internal Medicine, Allergy and Clinical Immunology Unit, Izmir, Turkey \\ ${ }^{6}$ Ege University, Faculty of Medicine, Department of Neurology, Izmir, Turkey \\ ${ }^{7}$ Ankara University, Department of Chest Medicine, Immunology and Clinical Immunology Unit, Ankara, Turkey \\ ${ }^{7 i}$ Ankara University, Faculty of Medicine, Department of Neurology, Ankara, Turkey
}

\section{KEY WORDS}

antiepileptics; carbamazepine;

HLA-A allele; HLA-B allele; drug hypersensitivity; delayed reactions;

SJS; TEN; MPE

\section{Corresponding author \\ Suna Büyüköztürk \\ Istanbul University \\ Faculty of Medicine \\ Department of Allergy \\ and Clinical Immunology \\ Istanbul, Turkey \\ Phone: +90 2125231241 \\ E-mail: sbuyuk@istanbul.edu.tr}

Doi

10.23822/EurAnnACI.1764-1489.23

\begin{abstract}
Summary
Background and objective. Many studies have shown associations between $H L A B^{*}$ 15:02, HLA-A*31:01 and carbamazepine (CBZ)-induced delayed cutaneous hypersensitivity reactions. The aim of this study is to evaluate a possible association between delayed cutaneous reactions to antiepileptic drugs (AEDs) and certain HLA-A and HLA-B alleles in the Turkish population. Methods. The study consisted of 3 groups: Group I (reactive group) included the patients who had documented delayed cutaneous reactions to any antiepileptic drug. Group II (non-reactive group) included the patients who have been on antiepileptic treatment at least for three months without any adverse reactions. Group III consisted of healthy subjects. The $H L A-A$ and $B$ alleles were analyzed in all groups. Results. Forty patients (29 female) had experienced different hypersensitivity reactions due to AEDs: maculopapular exanthema (26 patients), Stevens-Johnson syndrome (6 patients), drug rash with eosinophilia and systemic symptoms (7 patients), toxic epidermal necrolysis (1 patient). Lamotrigine (11) and CBZ (10) were the most common culprit drugs involved in the reactions. The HLA-B*15:02 was not present in any of the study groups. However, $H L A-B * 35: 02$ was found in 4 patients from the reactive group, while it was not observed in non-reactive patients and was detected in only one healthy subject $(p=0.021)$. Conclusion. Although our preliminary results did not indicate a strong allele association with AED hypersensitivity, $H L A-B^{*}$ 35:02 appears to be a candidate allele for MPE / DRESS / DIHSS induced by AED's in Turkish population. Further studies with a larger sample size may result in more comprehensive data about the genetic tendency for AED hypersensitivity in the Turkish population.
\end{abstract}




\section{Introduction}

Drug hypersensitivity entails an important clinical morbidity. It consists of a variety of phenotypes, mainly the cutaneous adverse reactions that range from milder skin reactions (e.g., exanthem, urticaria, and angioedema) to severe cutaneous adverse reactions (SCARs) (1). SCARs are life-threatening, and include Stevens-Johnson syndrome (SJS), toxic epidermal necrolysis (TEN), and drug reaction with eosinophilia and systemic symptoms (DRESS) or drug-induced hypersensitivity syndrome (DIHS) (2). These reactions are differed from other drug reactions by their high morbidity and mortality rates. At this point, any factors that can predict the development of these particular reactions are highly warranted. So far, a limited number of drugs are associated with a high risk for SJS and TEN: several antiepileptic agents, especially CBZ, phenytoin, phenobarbital and lamotrigine, antibacterial sulfonamides, anti-inflammatory drugs of the oxicam family, allopurinol and the antiretroviral agent nevirapine (3).

Over the last decade, a large number of studies have shown associations between various HLA alleles and different delayed drug hypersensitivities (4). To date, the best characterized HLA-associated drug related hypersensitivity reactions are documented by abacavir, nevirapine, $\mathrm{CBZ}$ and allopurinol (5). Among these drugs, abacavir has the strongest association with HLA-B*57:01 allele. Individuals with this allele have approximately a $50 \%$ chance of developing abacavir hypersensitivity syndrome, while no one without this allele is predicted to develop an immunologically confirmed hypersensitivity reaction, whereas $\mathrm{CBZ}$ hypersensitivity has been shown to be strongly associated with the HLA-B*15:02 allele (6). The latter association was first demonstrated in Han Chinese patients, in which 44 with CBZ-induced SJS/TEN were positive for HLA-B* 15:02 compared to $3 \%$ in the CBZ-tolerant cohort (odds ratio 2504) (7). Subsequent studies have also confirmed this association in Thai, Korean, Malaysian and Indian populations (8). As a result of this suggestive results, the US Food and Drug Administration has recommended prescreening testing for HLA-B*15:02 allele before prescribing CBZ in the ethnic groups at risk (9).

Later on another allele, HLA-A*31:01 was reported to be associated with all phenotypes of CBZ-induced cutaneous reactions, including maculopapular exanthema (MPE), DRESS and SJS/TEN in European and Japanese populations $(10,11)$.

So far, an association of certain HLA types with delayed drug hypersensitivity reactions has not been studied in Turkish population. So, we aimed to investigate whether any HLA A or B based genetic tendency exists for AED induced hypersensitivity reactions in Turkish population.

\section{Methods}

This was a multi-centered, case-control study in which the Neurology and Allergy Immunology Departments of 5 Universities from 4 different regions of Turkey, Istanbul, Ankara (two centers), Izmir and Rize have participated. The study population involved three groups as below:

Group 1, patients with documented delayed cutaneous drug reactions (CDRs) due to any AED (CBZ, oxcarbazepine, phenytoin or lamotrigine) determined by file screenings, and the patients who experienced delayed CDRs during the follow up period of three months after the starting of a newly prescribed AED.

Group 2, the patients who have been receiving treatment with any AED at least for three months without any drug related reactions (drug taking controls).

Group 3, healthy subjects with no drug hypersensitivity reaction and with no use of any AED.

The clinical features of the past drug reactions recorded on patient files were evaluated by expert dermatologists. Newly emerging CDRs were classified by the same experts according to the following criteria (14). SJS was diagnosed if a rapidly developing blistering exanthema of purpuric macules and target-like lesions accompanied by mucosal involvement with skin detachment of up to $10 \%$ of the body surface area were present. Patients with skin detachment from 10 to $29 \%$ of the body surface area were categorized as SJS-TEN overlap, whereas TEN was defined by widespread macules or blisters with skin detachment of up to $30 \%$ of the body surface area. The inclusion criteria of DRESS were a suspected drug reaction with MPE, plus the involvement of at least one internal organ (e.g., hepatitis, pneumonitis, myocarditis, pericarditis, nephritis), one of either lymphadenopathy or hematologic abnormality (e.g., eosinophilia, atypical lymphocytosis) and a fever above $38^{\circ} \mathrm{C}$. Patients who met three or more of these criteria were considered as DRESS (14). The patients who were exposed to another "high-risk" drug or had any viral or bacterial infection in the same period when drug hypersensitivity symptoms arose, were excluded from the study.

The patients determined by file screenings were called and invited to participate to the study. Twenty-eight patients accepted to be involved and give blood samples. Also, twelve patients diagnosed as AED related CDR during their follow-up visits volunteered to be study subjects.

The ethical approval was obtained from Ethical Committee of Istanbul Medical Faculty, Istanbul University. All volunteer participants gave written informed consents prior to enrollment on the study.

\section{HLA analysis}

$10 \mathrm{ml}$ blood samples were collected in EDTA tubes from all groups. All the samples were genotyped at the Department of Medical Biology of Istanbul Medical Faculty, which has accreditation to perform clinical HLA typing by the European Federation of Immunogenetics.

The genomic DNAs were obtained from peripheral venous blood samples by using the Bio-robot EZ1 (Qiagen N.V., Venlo, 
The Netherlands). Genotyping of HLA-B allele was performed by the PCR-SSOP method (polymerase chain reaction with sequence specific oligonucleotide probe) using Lambda One subtype kits (PCR-SSP, InGen, Technopolis, Chilly Mazarin, France) and confirmed by Olerup SSP high resolution HLA-B kits (PCR-SSP, Geno-Vision Inc, West Chester, Pennsylvania, USA). HLA-A alleles were typed by SBT method by Invitrogen kits (Thermo Fisher Scientific, Waltham, Mass, USA).

\section{Statistical analysis}

Statistical analyses were performed by Statistical Package for Social Sciences (SPSS 21.0) for Windows. Numeric values were expressed as mean $\pm \mathrm{SEM}$, whereas nominal values were given as $\mathrm{n}(\%)$. Comparisons of the frequencies of HLA-A and HLA-B alleles between the subgroups were performed using Fisher's exact tests. All P-values were two-tailed. The statistical significance was defined as $\mathrm{P}<0.05$.

\section{Results}

The age and sex distribution were comparable in all study groups except Group II, in which the rates of female and male were equal (Table I). In the reactive group, the most common drugs causing CDR were lamotrigine ( $\mathrm{n}=11)$, CBZ ( $\mathrm{n}: 10)$, and different combinations of these drugs (11). Other drugs were phenytoin $(\mathrm{n}=$ $3)$, valproate $(\mathrm{n}=1)$, prymidone (1), gababentin (1) pregabalin (1), topiramate (1). Most of the reactions were MPE $(n=26)$ (Table II). The numbers of serious AED reactions were 6 for SJS, 1 for TEN and 7 for DRESS / DIHSS. Lamotrigine was the sole responsible drug (in 3 patients) or as a combination with another antiepileptic drug (in 3 additional patients) in patients presenting with SJS (Table III). On the other hand, CBZ was more frequent in DRESS / DIHSS (Table IV). Allele frequencies were similar in both AED reactive and tolerant patients (Table $\mathbf{V}$ and Table $\mathbf{V I}$ ). However, the HLA-B*35:02 was found in 4 of 40 AED-reactive patients (in two patients with MPE and two patients with DRESS

Table I - Age and gender characteristics of the study groups.

\begin{tabular}{llll}
\hline & Group 1 $(\mathbf{n}=\mathbf{4 0})$ & Group 2 $(\mathbf{n}=\mathbf{6 0})$ & Group 3 $(\mathbf{n}=\mathbf{1 1 8})$ \\
\hline age $($ mean \pm SEM $)(\min -\max )$ & $32.6 \pm 13.8(18-74)$ & $36.2 \pm 12.6(20-64)$ & $34.3 \pm 11.7(21-70)$ \\
\hline sex $($ female / male $)$ & $29 / 11$ & $30 / 30$ & $85 / 33$ \\
\hline
\end{tabular}

Table II - The characteristics of the patients with antiepileptic drug induced hypersensitivity reactions.

\begin{tabular}{lcccc}
\hline Phenotypes & SJS / TEN & DRESS / DIHSS & MPE & FDE \\
\hline $\mathrm{N}$ & $6(1$ TEN) & 7 & 26 & 1 \\
sex (female / male) & $6 / 0$ & $3 / 4$ & $20 / 6$ & $\mathrm{M}$ \\
age (mean \pm SEM) & $37.7 \pm 19.9$ & $28 \pm 8.9$ & $32.9 \pm 13.1$ & 54 \\
(min - max) & $(21-74)$ & $(19-44)$ & $(16-62)$ & \\
\hline
\end{tabular}

Abbreviations: SJS, Stevens-Johnson syndrome; TEN, toxic epidermal necrolysis, DRESS, drug rush with eosinophilia and systemic symptoms; DIHSS, drug induced hypersensitivity syndrome; MPE; maculopapular exanthema; FDE; fixed drug eruption.

Table III - The HLA-B and HLA-A alleles of 5 patients with SJS and 1 TEN induced by antiepileptic drugs (DNA sampling could not be done in one SJS patient because the blood sample was clotted).

\begin{tabular}{|c|c|c|c|c|c|}
\hline \multirow{2}{*}{$\begin{array}{l}\text { Patients } \\
\mathrm{SB}\end{array}$} & \multicolumn{2}{|c|}{ HLA-B alleles } & \multicolumn{2}{|c|}{ HLA-A alleles } & \multirow{2}{*}{$\begin{array}{l}\text { Culprit antiepileptic } \\
\text { lamotrigine + valproate }\end{array}$} \\
\hline & 07:05 & 44:02 & 02:01 & $24: 02$ & \\
\hline MV & $15: 01$ & $35: 01$ & 02:01 & $24: 02$ & lamotrigine \\
\hline ÇK & $40: 02$ & $51: 01$ & $11: 01$ & $32: 01$ & lamotrigine + topiramate \\
\hline GY & $38: 01$ & 49:01 & 01:01 & 03:01 & lamotrigine \\
\hline MG & $35: 03$ & $35: 03$ & $24: 02$ & $32: 01$ & lamotrigine + oxcarbazepine \\
\hline PB (TEN) & $27: 07$ & $51: 01$ & $24: 02$ & $24: 03$ & carbamazepine + phenytoin \\
\hline
\end{tabular}

Abbreviations: SJS, Stevens-Johnson syndrome; TEN, toxic epidermal necrolysis. 
Table IV - The HLA-B and HLA-A alleles of 7 patients with DRESS / DIHSS induced by antiepileptic drugs. $B^{*} 35: 02$ allele was present in two more patients with MPE.

\begin{tabular}{ccccll}
\hline Patients & \multicolumn{2}{c}{ HLA-B alleles } & \multicolumn{2}{c}{ HLA-A alleles } & Culprit Antiepileptics \\
\hline FY & $18: 01$ & $35: 08$ & $24: 02$ & $32: 01$ & pyrimidone \\
\hline FK & $15: 17$ & $53: 01$ & $02: 01$ & $68: 02$ & lamotrigine \\
\hline NÇ & $07: 02$ & $51: 01$ & $11: 01$ & $24: 02$ & carbamazepine \\
\hline PÇ & $08: 01$ & $51: 01$ & $01: 01$ & $26: 01$ & carbamazepine \\
\hline NÖ & $27: 04$ & $\mathbf{3 5 : 0 2}$ & $03: 01$ & $24: 02$ & pregabaline \\
\hline SB & $\mathbf{3 5 : 0 2}$ & $51: 01$ & $31: 01$ & $68: 01$ & carbamazepine \\
\hline ŞB & $44: 03$ & $51: 01$ & $02: 01$ & $03: 01$ & carbamazepine \\
\hline
\end{tabular}

DRESS, drug rush with eosinophilia and systemic symptoms; DIHSS, drug induced hypersensitivity syndrome; MPE; maculopapular exanthema.

/ DIHSS) while it did not exist in the AED tolerant group, and it was found in only one subject in the healthy control group (Table IV). This difference was statistically significant $(\mathrm{p}=0.021$, OR $10.48,95 \% \mathrm{CI}=1.205$ - 95.336).

In HLA-A evaluation, no predominant allele frequency was found in any of the groups. HLA-A* 31:01 existed in 3 of the AED reactants (one DRESS, two MPE) while it was found in 2 of the non-reactants $(\mathrm{p}=0.26)$. The HLA B*15:02 was not detected in any of the samples.

\section{Discussion}

This study is the first one to evaluate an association between AED hypersensitivity and HLA alleles in the Turkish population. Briefly, we did not find any specific HLA A or B alleles in delayed cutaneous reactions to AEDs. The results related to HLA-B*15:02 allele association in SJS to CBZ were in accordance with European studies. However, HLA-B*35:02, which has not been described as an associated marker with AED hypersensitivity in previous trials, was detected in our study group. HLA-B*15:02 allele is accepted as a strong marker for CBZ induced SJS / TEN in the patients with Asian descent. However, previous studies conducted in European countries showed that HLA-B*15:02 was a very rare allele in Caucasians, indicating that this marker cannot be used universally for CBZ related SJS $(12,13)$. Lonjou et al. also did not find the existence of an alternative single HLA-B allele with a very strong association in Europe, except for four patients with Asian ancestry who presented HLA-B*15:02. They proposed that different alleles at the same locus could be responsible for increased disease risk in different populations (13). Our country is located in the middle of Asia and Europe continents and the majority of the people living in Turkey are Caucasians, expectedly, our results were in accordance with European results.

Another genetic association proposed for AED-induced severe cutaneous reactions is $\operatorname{HLA}^{*} 31: 01$, which was first shown in CBZ related MPE and DRESS in Han Chinese (14). Later, HLA-A*31:01 was reported to associate with all phenotypes of CBZ-induced cutaneous reactions, including MPE, DRESS and SJS / TEN in Europeans and Japanese $(10,11)$. More recently, it was demonstrated that HLA-A*31:01 was only associated with CBZ-DRESS but not with CBZ-SJS / TEN in Europeans or Chinese (15). The authors found that HLA-A*31:01 had a sensitivity of $70 \%$ and a specificity of $96 \%$ as a predictor for CBZDRESS in Europeans, yet had a sensitivity of $50 \%$ and a specificity of $96 \%$ in Chinese. In our series, we observed HLA*31:01 in 3 of 40 AED reactive patients (one of 7 DRESS / DHSS and 2 of $26 \mathrm{MPE}$ ) and 2 of 60 tolerant patients (not-significant). Accordingly, HLA*31:01 does not seem to be related with DRESS / DHSS or SJS in our study patients. However, this result needs to be cautiously evaluated as the number of the patients presenting with DRESS / DHSS in our group is very limited.

As similar to our results, in another study conducted in patients of European origin, no single major HLA-related genetic risk factor was identified for lamotrigine-induced SCARs (15). The authors obtained only suggestive evidence for $B^{*} 58: 01$, $\mathrm{A}^{*}$ 68:01, $\mathrm{Cw}^{*} 07: 18, \mathrm{DQB} 1^{*} 06: 09$, and $\mathrm{DRB}^{*} 13: 01$. We detected $\mathrm{B}^{*}$ 58:01 in only two patients with MPE (one with lamotrigine, the second with CBZ). Although 4 reactive and 2 non-reactive patients presented HLA-A ${ }^{*} 68: 01$, this difference was not significant. But interestingly, one patient with DRESS caused by CBZ displayed $A^{*} 68: 01$ allele together with $A^{*} 31: 01$. The possibility that this special haplotype may reflect a risk for DRESS needs further exploration in a larger sample series.

Apart from the previously reported HLA alleles in these particular reactions, we found a possible new HLA allele association in delayed type cutaneous reactions to AEDs. In this sense HLA-B*35:02, which has not been described as an associated marker with AED hypersensitivity in previous trials, was detected in four patients of the AED reactive group, while it was absent in AED tolerant patients, and was present in only one of healthy subjects. Although 
Table $\boldsymbol{V}$ - HLA alleles of antiepileptic reactive patients.

\begin{tabular}{|c|c|c|c|c|}
\hline & HLA-B & HLA-B & HLA-A & HLA-A \\
\hline FK & $15: 17$ & $53: 01$ & 02:01 & $68: 02$ \\
\hline $\mathrm{FA}$ & $55: 01$ & $55: 01$ & 03:01 & $24: 02$ \\
\hline VA & $38: 01$ & 53:01 & 01:01 & $24: 02$ \\
\hline $\mathrm{FY}$ & $18: 01$ & $35: 08$ & 24:02 & $32: 01$ \\
\hline TK & $51: 01$ & $57: 01$ & 01:01 & 01:01 \\
\hline SB & $07: 05$ & $44: 02$ & 02:01 & $24: 02$ \\
\hline $\mathrm{NÇ}$ & 07:02 & $51: 01$ & 11:01 & $24: 02$ \\
\hline ABK & $18: 01$ & $35: 03$ & 01:01 & 02:01 \\
\hline PÇ & 08:01 & $51: 01$ & 01:01 & $26: 01$ \\
\hline NÖ & $27: 04$ & $35: 02$ & 03:01 & $24: 02$ \\
\hline $\mathrm{DE}$ & $35: 01$ & $50: 01$ & 02:02 & 03:01 \\
\hline E T & 08:01 & $47: 01$ & 01:01 & 01:01 \\
\hline MV & $15: 01$ & $35: 01$ & 02:01 & $24: 02$ \\
\hline AT & $40: 02$ & $51: 01$ & 03:01 & $24: 02$ \\
\hline ÇK & $44: 02$ & $55: 01$ & 11:01 & $32: 01$ \\
\hline SB & $35: 02$ & $51: 01$ & $31: 01$ & $68: 01$ \\
\hline MÜ & $38: 01$ & $58: 01$ & 26:01 & $33: 03$ \\
\hline $\mathrm{HG}$ & $44: 02$ & $47: 01$ & 02:01 & 03:xx \\
\hline $\mathrm{HL}$ & $50: 01$ & $51: 01$ & 02:01 & $24: 02$ \\
\hline $\mathrm{E}$ & $15: 01$ & $51: 01$ & 02:01 & $24: 02$ \\
\hline NA & $35: 01$ & $35: 03$ & 02:01 & $68: 01$ \\
\hline GY & $38: 01$ & 49:01 & 01:01 & 03:01 \\
\hline MG & $35: 03$ & $35: 03$ & $24: 02$ & $32: 01$ \\
\hline KK & $14: 02$ & $35: 02$ & 02:01 & 03:01 \\
\hline SK & $15: 01$ & $15: 01$ & 02:01 & 03:02 \\
\hline $\mathrm{AD}$ & $35: 03$ & $50: 01$ & 02:05 & $31: 01$ \\
\hline BM & $07: 05$ & $58: 01$ & 03:01 & 03:02 \\
\hline SY & $35: 02$ & $44: 02$ & 24:02 & $31: 01$ \\
\hline YS & $40: 02$ & $51: 01$ & 02:01 & $68: 01$ \\
\hline AV & $51: 01$ & $55: 01$ & 02:01 & $24: 02$ \\
\hline $\mathrm{PB}$ & $27: 07$ & $51: 01$ & 24:02 & $24: 03$ \\
\hline $\mathrm{AK}$ & 07:02 & $57: 01$ & $30: 01$ & $30: 01$ \\
\hline SK & $53: 01$ & $55: 02$ & 02:01 & 03:01 \\
\hline HT & $18: 01$ & $35: 01$ & $02: 17$ & $03: 02$ \\
\hline ŞB & $44: 03$ & $51: 01$ & 02:01 & 03:01 \\
\hline MK & $40: 01$ & $44: 02$ & 02:01 & $24: 02$ \\
\hline SD & $35: 03$ & $35: 08$ & 01:01 & $24: 02$ \\
\hline $\mathrm{ND}$ & $15: 03$ & $41: 01$ & 01:01 & $02: 05$ \\
\hline $\mathrm{DH}$ & $15: 18$ & $35: 01$ & 03:01 & 68:01 \\
\hline ES & $13: 01$ & $55: 01$ & 11:01 & $30: 01$ \\
\hline
\end{tabular}

Table VI - HLA alleles of antiepileptic tolerant patients.

\begin{tabular}{|c|c|c|c|c|}
\hline Case & HLA-B & HLA-B & HLA-A & HLA-A \\
\hline MY & $38: 01$ & $44: 02$ & 03:02 & $26: 01$ \\
\hline $\mathrm{EE}$ & 08:01 & $38: 01$ & 01:01 & $11: 01$ \\
\hline $\mathrm{NZ}$ & $35: 01$ & $58: 01$ & $11: 01$ & $31: 01$ \\
\hline GÇ & 08:01 & $13: 02$ & 01:01 & $30: 01$ \\
\hline ÜG & 08:01 & $35: 01$ & $01: 01$ & 03:01 \\
\hline ÜA & $07: 05$ & $55: 01$ & 02:01 & $26: 01$ \\
\hline GÜ & $41: 01$ & 51:01 & $24: 02$ & $32: 01$ \\
\hline KT & $35: 01$ & $55: 01$ & 03:01 & $24: 02$ \\
\hline ŞÇ & $15: 01$ & 53:01 & $30: 02$ & 68:01 \\
\hline $\mathrm{KA}$ & 07:02 & 08:01 & 03:01 & 30:01 \\
\hline BAT & $27: 03$ & $44: 03$ & 02:01 & $33: 01$ \\
\hline KES & $40: 01$ & $41: 01$ & 02:01 & 02:02 \\
\hline $\mathrm{CE}$ & $27: 02$ & $55: 01$ & 01:01 & $24: 02$ \\
\hline ME & 07:02 & 51:01 & $02: 17$ & $24: 02$ \\
\hline SY & $14: 02$ & $55: 01$ & $24: 02$ & $33: 01$ \\
\hline ST & 51:01 & 51:01 & 01:01 & 11:01 \\
\hline $\mathrm{NU}$ & 50:01 & $51: 01$ & 01:01 & 02:01 \\
\hline ÖK & $35: 03$ & 49:01 & 02:01 & $24: 02$ \\
\hline $\mathrm{HS}$ & 13:01 & $51: 09$ & $32: 01$ & 68:01 \\
\hline Mİ & 07:02 & $35: 01$ & 03:01 & 11:01 \\
\hline EY & $44: 03$ & $51: 01$ & 02:01 & 02:01 \\
\hline MÖ & $13: 02$ & $35: 03$ & $11: 01$ & 11:01 \\
\hline HBB & 38:01 & $40: 01$ & $25: 01$ & $32: 01$ \\
\hline MY & 08:01 & $18: 01$ & 03:02 & $32: 01$ \\
\hline AA & $14: 02$ & $58: 01$ & $11: 01$ & $33: 01$ \\
\hline $\mathrm{CU}$ & $40: 01$ & $55: 01$ & 02:01 & 02:01 \\
\hline ET & $13: 02$ & $27: 03$ & 02:01 & $30: 01$ \\
\hline AKA & $37: 01$ & $39: 01$ & 01:01 & $26: 01$ \\
\hline HSE & $44: 03$ & $51: 01$ & 01:01 & $29: 02$ \\
\hline LU & $44: 03$ & $58: 01$ & 01:01 & 02:01 \\
\hline $\mathrm{FU}$ & $35: 01$ & $38: 01$ & 01:01 & 03:02 \\
\hline MA & 07:02 & $51: 01$ & 02:01 & $24: 02$ \\
\hline GG & $18: 01$ & $44: 02$ & $11: 01$ & $24: 02$ \\
\hline $\mathrm{KD}$ & 13:01 & $51: 01$ & 02:01 & $30: 01$ \\
\hline MA & $27: 02$ & $51: 01$ & $02: 01$ & 26:01 \\
\hline SK & 07:02 & $44: 03$ & 02:01 & $33: 03$ \\
\hline ÖK & 51:01 & $57: 01$ & 01:01 & $24: 02$ \\
\hline ÖE & $51: 01$ & $55: 01$ & $26: 01$ & $26: 01$ \\
\hline YB & $35: 01$ & $35: 01$ & 03:01 & 03:01 \\
\hline GT & $35: 03$ & $55: 01$ & 03:01 & 24:02 \\
\hline SÇ & 08:01 & $40: 01$ & $26: 01$ & 26:01 \\
\hline SA & 07:02 & $14: 02$ & $11: 01$ & $33: 01$ \\
\hline
\end{tabular}


Table VI - HLA alleles of antiepileptic tolerant patients.

\begin{tabular}{ccccc}
\hline FZÇ & $44: 02$ & $57: 01$ & $02: 01$ & $24: 02$ \\
\hline NG & $35: 01$ & $44: 02$ & $02: 01$ & $03: 01$ \\
\hline MD & $50: 01$ & $51: 01$ & $02: 01$ & $26: 01$ \\
\hline BM & $35: 03$ & $35: 03$ & $24: 02$ & $33: 03$ \\
\hline YD & $48: 01$ & $52: 01$ & $11: 01$ & $24: 02$ \\
\hline GY & $35: 03$ & $39: 06$ & $03: 01$ & $31: 01$ \\
\hline HY & $08: 01$ & $18: 01$ & $24: 02$ & $30: 02$ \\
\hline İS & $35: 01$ & $51: 01$ & $02: 01$ & $24: 02$ \\
\hline AFM & $38: 01$ & $38: 01$ & $03: 01$ & $33: 03$ \\
\hline İA & $38: 01$ & $38: 01$ & $26: 01$ & $26: 01$ \\
\hline EB & $44: 02$ & $48: 01$ & $24: 02$ & $26: 01$ \\
\hline EG & $08: 01$ & $14: 02$ & $30: 02$ & $33: 01$ \\
\hline EA & $51: 01$ & $58: 01$ & $02: 01$ & $02: 01$ \\
\hline EÇ & $35: 01$ & $40: 01$ & $02: 01$ & $24: 02$ \\
\hline KK & $15: 01$ & $38: 01$ & $02: 01$ & $11: 01$ \\
\hline PK & $14: 02$ & $18: 01$ & $33: 01$ & $66: 01$ \\
\hline MMÖ & $51: 01$ & $55: 01$ & $01: 01$ & $02: 01$ \\
\hline HP & $07: 02$ & $35: 01$ & $02: 01$ & $02: 01$ \\
\hline
\end{tabular}

this association seems to be inspiring, the results to be derived from more patients are needed to draw more definite conclusion and whether or not this HLA allele is really associated with certain type of reaction with certain AEDs in Turkish population.

We have some limitations in this study. Both small sample size for each SCAR and the heterogeneity of the responsible AEDs were the main limiting factors. The many previous studies on CBZ hypersensitivity reactions suffered from the low incidences, difficulty of patient's enrollment, and small sample sizes (17). Focusing on a specific drug and analyzing HLA genotype-phenotype correlations in the clinical spectrum, could provide more comprehensive pharmacogenetic data in AED-induced hypersensitivity reactions. So far, there is no information about the frequency of severe drug reactions to antiepileptic drugs in the Turkish population. Accordingly, in this preliminary study the target drug and the number of centers needed to provide a sufficient sample size could not be predicted.

In conclusion, the results of this preliminary study demonstrated that any HLA-A or B allele was not strongly associated with cutaneous adverse reactions to AEDs in the study patients. The weak HLA*35:02 association needs to be clarified. Therefore, further studies with a larger sample size would be helpful in analyzing genetic tendency for AED hypersensitivity in the Turkish population.

\section{Conflict of interest}

The authors declare that they have no conflict of interest.

\section{Acknowledgments}

This study was supported by the Research Found of the Istanbul University. Project no: 36857.

\section{References}

1. Phillips EJ, Chung WH, Mockenhaupt M, et al. Drug hypersensitivity: pharmacogenetics and clinical syndromes. J Allergy Clin Immunol 2011; 127:S60-6.

2. Lonjou C, Borot N, Sekula P, et al. A European study of HLA-B in Stevens-Johnson syndrome and toxic epidermal necrolysis related to five high-risk drugs. Pharmacogenet Genomics 2008; 18:99-107.

3. Roujeau JC, Stern RS. Severe adverse cutaneous reactions to drugs. N Engl J Med 1994; 331:1272-85.

4. Pirmohamed M, Ostrov DA, Park BK. New genetic findings lead the way to a better understanding of fundamental mechanisms of drug hypersensitivity. J Allergy Clin Immunol 2015; 136:236-44.

5. Pavlos R, Mallal S, Phillips EJ. HLA and pharmacogenetics of drug hypersensitivity. Pharmacogenomics 2012; 13:1285-306.

6. Yun J, Adam J, Yerly D, et al. Human leukocyte antigens (HLA) associated drug hypersensitivity: consequences of drug binding to HLA. Allergy 2012; 67:1338-46.

7. Chung WH, Hung SI, Hong HS, et al. Medical genetics: a marker for Stevens-Johnson syndrome. Nature 2004; 428:86.

8. Tangamornsuksan W, Chaiyakunapruk N, Somkrua R, et al. Relationship between the HLA-B/1502 allele and carbamazepine-induced Stevens-Johnson syndrome and toxic epidermal necrolysis: a systematic review and meta-analysis. JAMA Dermatol 2013; 149:1025-32.

9. US Food and Drug Administration. Information for healthcare professionals: dangerous or even fatal skin reactions - carbamazepine (marketed as Carbatrol, Equetro, Tegretol, and generics). Available at: https://www.fda.gov/Drugs/DrugSafety/PostmarketDrugSafetyInformationforPatientsandProviders/ucm 124718.htm; 2007.

10. McCormack M, Alfirevic A, Bourgeois S, et al. HLA-A*3101 and carbamazepine-induced hypersensitivity reactions in Europeans. N Engl J Med 2011; 364:1134-43.

11. Ozeki T, Mushiroda T, Yowang A, et al. Genome-wide association study identifies HLA-A*3101 allele as a genetic risk factor for carbamazepine-induced cutaneous adverse drug reactions in Japanese population. Hum Mol Genet 2011; 20:1034-41.

12. Alfirevic A, Jorgensen AL, Williamson PR, et al. HLA-B locus in Caucasian patients with carbamazepine hypersensitivity. Pharmacogenomics 2006; 7:813-8.

13. Lonjou C, Thomas L, Borot N, et al. A marker for Stevens-Johnson syndrome: ethnicity matters. Pharmacogenomics J 2006; 6:265-8.

14. Hung SI, Chung WH, Jee SH, et al. Genetic susceptibility to carbamazepine-induced cutaneous adverse drug reactions. Pharmacogenet Genomics 2006; 16:297-306.

15. Genin E, Chen DP, Hung SI, et al. HLA-A*31:01 and different types of carbamazepine-induced severe cutaneous adverse reactions: an international study and meta-analysis. Pharmacogenomics J 2014; 14:281-8.

16. Kazeem GR, Cox C, Aponte J, et al. High-resolution HLA genotyping and severe cutaneous adverse reactions in lamotrigine-treated patients. Pharmacogenet Genomics 2009; 19:661-5.

17. Hsiao YH, Hui RC, Wu T, et al. Genotype-phenotype association between HLA and carbamazepine-induced hypersensitivity reactions: strength and clinical correlations. J Dermatol Sci 2014; 73:101-9. 